\title{
Duración de los viajes migratorios de mexicanos indocumentados en Estados Unidos bajo la política de reforzamiento de la frontera
}

\author{
Ricardo Mora Téllez
}

\author{
Universidad de Princeton, Estados Unidos
}

\section{Resumen}

El artículo estudia el efecto que tiene la política de reforzamiento de la frontera sobre la duración de los viajes migratorios de los migrantes mexicanos indocumentados. En particular interesa analizar como el incremento de los riesgos y costos de cruce fronterizo clandestino, que surgen de esta política migratoria, afectan al tiempo de permanencia de los migrantes indocumentados en Estados Unidos. Para ello, se estimó un modelo probabilístico de riesgo en tiempo discreto logit, el cual permite determinar cambios en las probabilidades de retorno y, por lo tanto, cambios en las tendencias de las duraciones de los viajes migratorios. La base de datos empleada fue la Mexican Migration Proyect (MMP). El modelo arrojó coeficientes negativos en los costos y riesgos de cruce fronterizo clandestino en relación a la probabilidad de retorno.

Palabras clave: Migración indocumentada, migración de retorno, reforzamiento de la frontera.

Abstract

Duration of migratory trips of undocumented Mexicans in the United States under the border enforcement policy

This article studies the effect of political border enforcement on the duration of the migratory journeys of undocumented Mexican migrants. In particular we analyze the effect of increase of the risks and costs of illegal border crossing that arise from this migration policy over the trip duration of undocumented migrants in the United States. For this purpose a logit model (Discrete-Time Hazard Model) was fit, which allows to determine changes in the odds of return and, therefore changes in trends in migratory journeys durations. The database used is the survey Mexican Migration Project (MMP). The model has negative coefficients on the variables costs and risks of clandestine border crossing in regard to the probability of return.

Key words: Undocumented migration, return migration, border enforcement. 


\section{INTRODUCCIÓN}

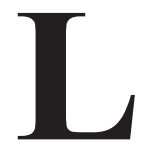

a migración México-Estados Unidos tienen una larga historia que puede ser dividida en fases o eras que se diferencian por las políticas migratorias implementadas por Estados Unidos y las condiciones legales de cruce fronterizo que prevalecen. El año de 1965 marca un nuevo cambio en la política migratoria estadounidense y da inicio a una nueva era (Massey et al., 2009). Ésta se caracteriza por la cancelación del Programa Bracero en 1964, la cual cerró o estrechó los canales autorizados de la migración mexicana a Estados Unidos y provocó una verdadera explosión de desplazamientos irregulares y la configuración de un nuevo modelo basado en la circulación de trabajadores indocumentados (Tuirán y Ávila, 2010). Durante los 21 años que duró este periodo de migración indocumentada, el gobierno de Estados Unidos operó de facto un programa de trabajadores invitados ya que sólo fueron asignados al reforzamiento de la frontera los recursos y el personal necesarios para aparentar que la frontera estaba bajo control y así tranquilizar a la opinión pública. Es decir, el costo de cruzar la frontera fue aumentado hasta el punto en el cual se logró alguna selectividad en el flujo migratorio, pero nunca aumentó lo suficiente como para obstaculizar seriamente la entrada de trabajadores mexicanos a Estados Unidos. De esta manera, los empleadores seguían teniendo acceso a la mano de obra barata de los migrantes mexicanos indocumentados, mientras que los ciudadanos americanos estaban convencidos de que la frontera se mantenía bajo control (Alba, 2007; Massey et al., 2002).

Bajo este escenario, si un migrante indocumentado era detenido durante su cruce fronterizo clandestino simplemente volvía a intentar cruzar la frontera cuantas veces fueran necesarias hasta que lograba su cometido. Este proceso fue llamado el modelo de pruebas repetitivas, "Repeated Trials Model" (Espenshade, 1990), el juego del gato y el ratón, "A Game of Cat-and-Mouse" (Koussoudji, 1992), o bien una puerta giratoria "Revolving Door" (Durand, 1994) (Massey et al., 2002).

Posteriormente en octubre de 1986, el congreso de Estados Unidos aprobó la ley "Immigration Reform and Control Act" (IRCA), que fue una reformulación de la política de inmigración con el objetivo de regularizar el estatus migratorio de millones de migrantes indocumentados que se encontraban en ese país y de controlar los cruces fronterizos indocumentados 
(Massey y Liang, 1989). Es a partir de esta ley que se empieza a configurar la política migratoria de control fronterizo, al autorizar un incremento inmediato de 50 por ciento en el presupuesto del Servicio de Naturalización e Inmigración (INS por sus siglas en ingles) para la vigilancia de la frontera (Massey et al., 2009).

Más adelante la política de reforzamiento de la frontera "Border Enforcement" surge formalmente en enero de 1993, a partir del estudio realizado para el INS denominado "Systematic Analysis of the Southwest Border", el cual fue elaborado por una empresa que desarrolla tecnología militar (Sandia National Laboratories). Este estudio recomienda a la patrulla fronteriza centrar sus esfuerzos en prevenir la entrada de personas indocumentadas, mediante la disuasión en vez de que trate de aprehenderlas al interior del país (Prevention Through Deterrence). Este reporte recomendó varias medidas para incrementar la dificultad de entrar al país, de las que destacan: la construcción de muros a lo largo de la frontera en las zonas urbanas que habitualmente utilizan los migrantes para internarse de manera subrepticia en Estados Unidos, aumentar el número de oficiales de la patrulla fronteriza y el uso de tecnología militar de vigilancia (radares, instrumentos de visión nocturna, detectores de movimiento, aviones no tripulados, helicópteros entre otros) (Tuirán y Ávila, 2010; Cornelius, 2007; Andreas, 1999). De ahí que Dunn (1997) y Nagengast (1998) hablen de una política de militarización de la frontera la cual implementa la doctrina castrense denominada conflicto de baja intensidad (Low-Intensity Conflict).

Bajo este enfoque se han desplegado en las áreas urbanas donde el cruce fronterizo de los migrantes indocumentados era más visible la operación Hold-the-Line en El Paso, Texas (1993), le siguieron la operación Gatekeeper en San Diego, California (1994), la operación Rio Grande en las ciudades Del Rio, Laredo y McAllen, al sur de Texas (1997) y la operación Safeguard en Nogales, Arizona (1999). Inicialmente estos programas se concentraron en estas ciudades disminuyendo el flujo migratorio indocumentado que transitaba por estos espacios, posteriormente todos estos operativos se han ampliado en distintas fases en los alrededores de estos puntos y en otras ciudades fronterizas, mientras que a lo largo de la frontera se dejaron sin fortificar remotas áreas rurales desérticas con condiciones climáticas extremas (Cornelius, 2007; Andreas, 1999). Como resultado los flujos migratorios han desviado su cruce fronterizo de los centros urbanos hacia zonas rurales cada vez más remotas y desérticas entre los segmentos fuertemente fortificados de la frontera. 
La lógica subyacente de esta operación fronteriza es disuadir los cruces fronterizos indocumentados al obligar a la migración no autorizada a cruzar la frontera a través de terrenos tan hostiles y peligrosos ${ }^{21}$ que quienes quisieran entrar sin la debida inspección desistan de su intento ya que los costos y los riesgos que deben asumir son muy elevados (Alba, 2010; Tuirán y Ávila, 2010; Cornelius, 2007; Orrenius, 2005; Massey et al., 2002; Andreas, 1999; Eschbach et al., 1999).

En relación a este nuevo ciclo migratorio, se ha observado un marcado desgaste de los mecanismos de circularidad migratoria y una mayor propensión a una migración de carácter más permanente (Alba, 2010; Ariza y Portes, 2010; Massey et al., 2009; Tuirán y Ávila, 2010). Esto se explica, en parte, porque la política de reforzamiento de la frontera ha aumentado los costos y los riesgos en el cruce fronterizo clandestino.

El artículo estudia el efecto que tiene la política de reforzamiento de la frontera sobre la duración de los viajes migratorios de los migrantes mexicanos indocumentados. En particular interesa analizar como el incremento en los riesgos y costos de cruce fronterizo clandestino, que surgen de esta política migratoria, afectan al tiempo de permanencia de los migrantes indocumentados en Estados Unidos. El trabajo se estructura de la siguiente manera. La primera sección es la introducción al estudio, la segunda analiza la manera en la cual la política de reforzamiento de la frontera ha incrementado los costos y riesgos de cruce fronterizo a los migrantes indocumentados, la tercera se enfoca al análisis del cambio del tiempo de permanencia de los migrantes indocumentados mexicanos en Estados Unidos bajo el periodo de reforzamiento de la frontera, la cuarta sección presenta el enfoque metodológico del estudio, mientras que la quinta presenta los resultados y la estimación de la probabilidad de retorno a lo largo del periodo de estudio, por último la sexta presenta las conclusiones.

\section{COSTOS DEL CRUCE FRONTERIZO CLANDESTINO}

Conforme el estricto reforzamiento de la frontera se ha implementado en los puntos de cruce fronterizo más fáciles y conocidos, el flujo migratorio indocumentado se ha desviado hacia puntos de entrada menos visibles entre montañas y desiertos, lejos de las áreas urbanas (Orrenius, 2004; An-

\footnotetext{
${ }^{21}$ Los migrantes que cruzan por estas zonas se exponen a condiciones ambientales que amenazan su vida. Por ejemplo, si intentan cruzar la frontera a través del desierto del Valle Imperial deben caminar como mínimo de 20 a 30 millas antes de llegar a un camino. En el verano, durante el día la temperatura media en este desierto es de $44.4^{\circ} \mathrm{C}$ y frecuentemente alcanza los $48.8^{\circ} \mathrm{C}$. Con lo cual, es físicamente imposible para los migrantes llevar suficiente agua para prevenir la deshidratación durante los dos días de caminata a través del desierto (Cornelius, 2001).
} 
dreas, 1999). Como consecuencia el cruce fronterizo es más peligroso y conlleva una mayor dificultad. Esto ha obligado a un número cada vez mayor de migrantes indocumentados a contratar el "servicio" de guía que ofrecen los contrabandistas de personas (conocidos como: "coyotes" o "polleros"), esto con el fin de que su entrada clandestina a través del desierto sea asistida (Massey et al., 2009; Cornelius, 2007). Como resultado, se observa que: conforme se intensifica el endurecimiento de la política migratoria de Estados Unidos la demanda de los servicios de los "coyotes" se incrementa, lo cual presiona al alza los precios que los contrabandistas de personas cobran a los migrantes (Alba, 2010; Massey et al., 2009; 2002; Tuirán y Ávila, 2010; Cornelius et al., 2008; Richter et al., 2007).

Aunado a esto, la desviación del cruce fronterizo hacia lugares cada vez más remotos también incrementa el precio de los servicios de los coyotes, ya que éstos deben incorporar los costos de transportar a los migrantes a puntos de cruce fronterizo distantes, así como los gastos que se derivan por albergar a los migrantes en localidades entre México y Estados Unidos, lo que aumenta los gastos operativos de cruzar la frontera de manera indocumentada (Massey y Riosmena, 2010; Massey et al., 2002; Cornelius, 2001).

Por otro lado, la promulgación por parte del gobierno de Estados Unidos de multas y sanciones penales más estrictas en contra de los traficantes de personas y los operativos de vigilancia en la frontera han provocado que las personas dedicadas a esta actividad ilícita enfrente un mayor riesgo de ser capturadas y sancionadas. Esta situación ha provocado que el precio que cobran los traficantes de personas a los migrantes aumente en compensación al riesgo que corren por prestar éstos servicios.

Por consiguiente, esta política migratoria además de incrementar a los "coyotes" sus costos operativos de pasar al otro lado a un migrante indocumentado también ha aumentado los riesgos de que sean sancionados por operar al margen de la ley. Como resultado tenemos que los traficantes de personas al ver que sus costos y riesgos se han incrementado éstos en compensación han aumentado el cobro por sus servicios. Es así como el endurecimiento de esta política migratoria en la práctica se ve reflejado como un "impuesto de cruce" a los migrantes indocumentados.

Asimismo, el costo que los "coyotes" cobran a los migrantes ha aumentado en los últimos años debido a que la delincuencia organizada ha impuesto un pago a los coyotes para permitirles trabajar en los territorios que controlan estos grupos delictivos (Izacara, 2014). 
La Figura 1 muestra el costo de contratación de los servicios de los "coyotes" pagados por los migrantes indocumentados de acuerdo al año en el cual cruzaron. En ella se aprecia un constante incremento en los costos de cruce a partir de 1993, año en el cual, da inicio la política de militarización de la frontera.

Figura 1: Costos promedio del pago a "Coyotes" (dólares reales a marzo de 2014), todos los puntos de cruce entre México y Estados Unidos

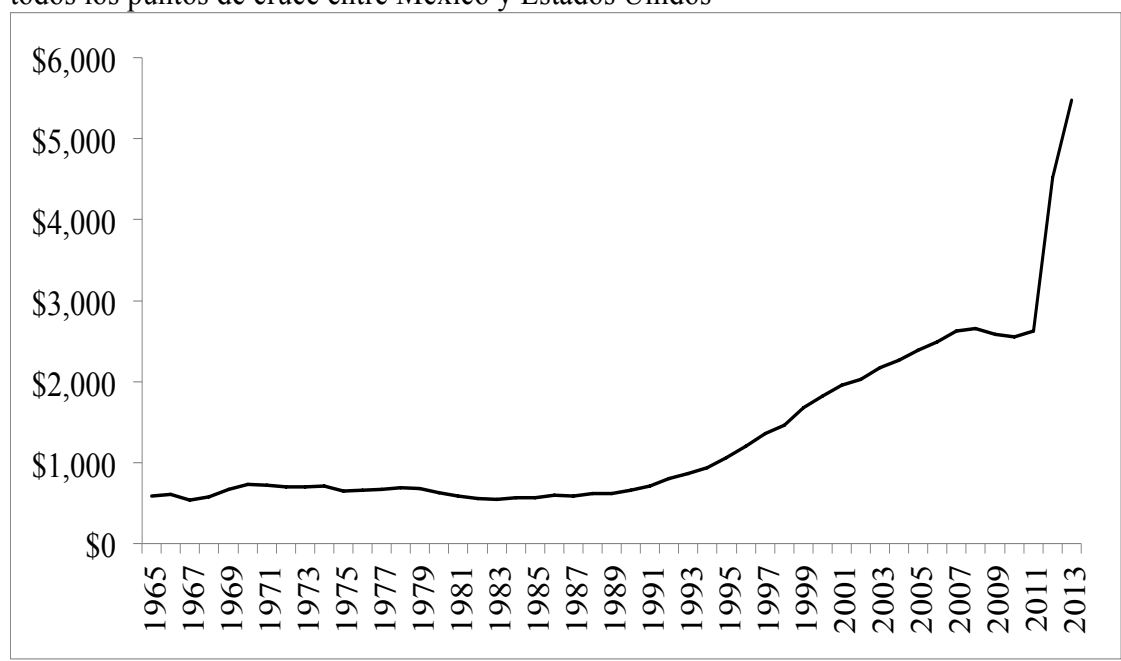

Fuente: elaboración propia con base en los datos de la encuesta MMP150 y de los datos del U.S. Department of Labor: Bureau of Labor Statistics Washington, D.C. Consumer Price Index of All Urban Consumers (CPI-U).

\section{Riesgos en el cruce fronterizo clandestino}

La política de reforzamiento de la frontera ha obligado a la migración no autorizada a cruzar la frontera a través de zonas desérticas e inhóspitas, consecuentemente el cruce fronterizo es más riesgoso debido a que durante la travesía los migrantes se exponen a terrenos hostiles bajo condiciones climáticas extremas (Massey y Riosmena 2010; Tuirán y Ávila, 2010; Cornelius, 2007; GAO, 2006; Orrenius, 2005; Massey et al., 2002; Alba, 2001; Eschbach et al., 2001; Eschbach et al., 1999).

En teoría, los migrantes deberían ser disuadidos de cruzar la frontera por el aumento en los riesgos y costos creados por la estrategia de reforzamiento de la frontera de Estados Unidos; sin embargo existen diferentes estudios que muestran que la política de "disuasión" no desalienta los cruces clandestino (Massey et al., 2009; 2002; Riosmena, 2009; Tuirán y Ávila, 2010; Cornelius et al., 2008; Andreas, 1999; Espenshade, 1994). Debido 
a que el ingreso esperado en Estados Unidos es a menudo entre ocho y diez veces mayor que el obtenido en México, la promesa de un empleo así como la consolidación de las redes migratorias familiares hacen que sea posible compensar los mayores riesgos y costos de un cruce clandestino (Alba, 2010; Cornelius et al., 2007; Massey et al., 2002). Por estos motivos, muchos migrantes si no logran ingresar a Estados Unidos en su primer intento simplemente realizan sucesivos intentos hasta que logran cruzar la frontera (Cornelius et al., 2007; Massey et al., 2002; Andreas, 2000; 1999; Espenshade, 1994).

Bajo esta estrategia, se ha observado una tendencia preocupante en el incremento de las muertes de los migrantes que ocurren durante el cruce fronterizo (Alba, 2010; Cornelius, 2006), ya que los migrantes al buscar evadir los controles fronterizos frecuentemente toman riesgos sustanciales, y algunos mueren en el proceso (Eschbach et al., 1999). Esto se ve reflejado en la tendencia de las muertes registradas durante los cruces fronterizos.

El reporte de la General Accounting Office (GAO) muestra que a finales de la década de 1980 y hasta principios de la década de 1990, el número de muertes en los pasos fronterizos tradicionales (zonas urbanas) declinó, no obstante en el periodo que abarca a finales de la década de 1990 y hasta el año 2005, el número de muertes se incrementó aproximadamente al doble de 241 muertes en 1999 a un total de 472 muertes registradas en 2005. La mayor parte del incremento de estas muertes ocurrió dentro del sector de la patrulla fronteriza de Tucson, el cual incluye gran parte del desierto de Arizona por donde se ha desviado el flujo migratorio (zonas rurales). Este aumento en las muertes ocurrió a pesar de que no hubo un incremento en el número de cruces indocumentados, lo que muestra que el riesgo asociado al cruce fronterizo clandestino se ha incrementado en los años recientes (GAO, 2006).

Basada en un estudio de la Organización Internacional para las Migraciones (OIM), la Figura 2 muestra las muertes registradas entre la frontera México-Estados Unidos durante el periodo que comprende de 1998 a 2013. Durante el cual, se observa que a partir del año 2005 en adelante, al menos un migrante al día pierde la vida tratando de cruzar la frontera. Con lo cual, una de las consecuencias de esta política ha sido un marcado incremento en el número de migrantes que mueren tratando de conseguir entrar a Estados Unidos (Tuirán y Ávila, 2010; Massey et al., 2009; 2002; Riosmena, 2009; Cornelius, 2006; 2004; Orrenius, 2004; Andreas, 1999; Eschbach et al., 1999). 
Figura 2: Muertes en la frontera entre México y Estados Unidos, 1998-2013

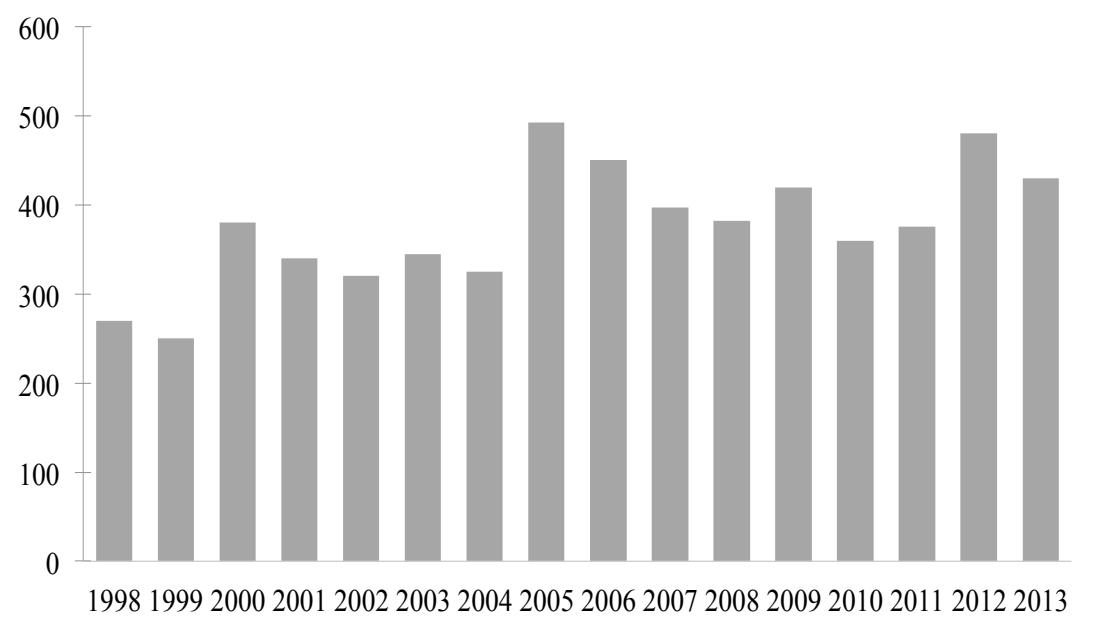

Fuente: International Organization for Migration (IOM), Fatal Journeys. Tracking Lives Lost during Migration, Geneva, Switzerland, 2014.

Además por cada una de las muertes, hay también muchas lesiones y serios percances que no son registrados (Massey et al., 2002). Esto se ve reflejado en un estudio realizado a 262989 migrantes aprehendidos por la Patrulla Fronteriza durante el periodo de diciembre de 1999 a mayo de 2000, el cual muestra que 70 por ciento de los migrantes se expusieron a algún tipo de riesgo físico durante su intento de cruzar la frontera de manera clandestina (Cornelius, 2001).

En un balance, la desviación de los flujos migratorios hacia zonas de cruce más riesgosas han incrementado la vulnerabilidad y los riesgos de accidentes y percances de todo tipo, incluido el de encontrar la muerte en la travesía 22 (Alba, 2007). Lo anterior muestra que las condiciones en el cruce rural han sido más peligrosas que las condiciones en el cruce urbano (Tuirán y Ávila, 2010; Cornelius et al., 2007; 2004; Massey et al., 2002; Eschbach et al., 2001; Andreas, 2000). De esta manera, el viejo juego del

\footnotetext{
${ }^{22}$ En una encuesta realizada por Cornelius levantada en dos comunidades rurales con altas tasa de emigración Tlacuitapa, Jalisco y Las Ánimas, Zacatecas, se encontró que ocho de cada diez personas piensa que a partir de la implementación de los operativos de reforzamiento de la frontera es mucho más peligroso el cruce de la frontera sin documentos, y cerca de dos terceras partes de los entrevistados conocen personalmente a alguien que ha muerto recientemente tratando de entrar clandestinamente a Estados Unidos (Cornelius, 2007).
} 
gato y el ratón entre los migrantes y la patrulla fronteriza ha sido llevado a un nuevo y más peligroso campo de juego (Eschbach et al., 2001). ${ }^{23}$

\section{Cambio en el tiempo de Permanencia de los migrantes INDOCUMENTADOS EN ESTADOS UNIDOS}

Para demostrar la conexión existente entre el aumento de los costos y riesgos asociados con el cruce fronterizo clandestino y el incremento del tiempo de permanencia de los migrantes indocumentados en Estados Unidos durante el periodo de reforzamiento de la frontera, se comparó, utilizando los datos de la encuesta Mexican Migration Project (MMP150), la tabla de sobrevivencia de los migrantes indocumentados de las siguientes cohortes de salida; Indocumentado (1965-1985), IRCA (1986-1993) y Reforzamiento de la Frontera (1994-2013). La Figura 3 muestra que en relación a la cohorte de salida del primer periodo de observación (1965-1985), el tiempo de permanencia de los migrantes indocumentados mexicanos en su primer viaje a Estados Unidos ha venido aumentando en las últimas dos cohortes de salida (1986-1993 y 1994-2013), ${ }^{24}$ observado un marcado incremento en el tiempo de permanencia de los migrantes que salieron del país durante la cohorte que corresponde al periodo de reforzamiento de la frontera (1994-2013).

De los resultados de la tabla de sobrevivencia se obtuvo la Tabla 1, la cual muestra los cuartiles del tiempo de sobrevivencia por cohorte de salida de los migrantes indocumentados. En ella se aprecia que 50 por ciento de los migrantes en su primer viaje presentó una mediana de 23 meses de permanencia durante la cohorte 1965-1985, aumentando a 36 meses en la cohorte 1986-1993, para posteriormente terminar con una mediana de 78 meses en la cohorte 1994-2013. Lo anterior representa un aumento en la mediana del tiempo de estancia en el periodo 1986-1993 de 56.5 por ciento con respecto a la primera cohorte de salida, mientras que para el periodo 1994-2013 representa un aumento de 116.7 por ciento en relación a la segunda cohorte de salida. Es decir, la tendencia creciente en el tiempo de permanencia de los migrantes se consolida en la cohorte 1994-2013, periodo que se caracteriza por el reforzamiento de la frontera por parte de Estados Unidos.

\footnotetext{
${ }^{23} \mathrm{Si}$ bien, los migrantes indocumentados centroamericanos que cruzan el territorio mexicano enfrentan diversos riesgos durante su paso por el territorio mexicano, estos riesgos no son analizados debido a que la población de estudio son los migrantes mexicanos indocumentados.

${ }^{24}$ Para probar homogeneidad entre cohortes, así como igualdad en la función de sobrevivencia, se estimaron la prueba de razón de verosimilitud (likelihood-ratio) y la prueba Logrank-test. En ambos casos, se rechazó la hipótesis nula al 95 por ciento de confianza.
} 
Figura 3: Proporción de migrantes indocumentados mexicanos que no han retornado de su primer viaje migratorio a Estados Unidos, según cohorte de salida (proporción estimada de supervivencia en meses)

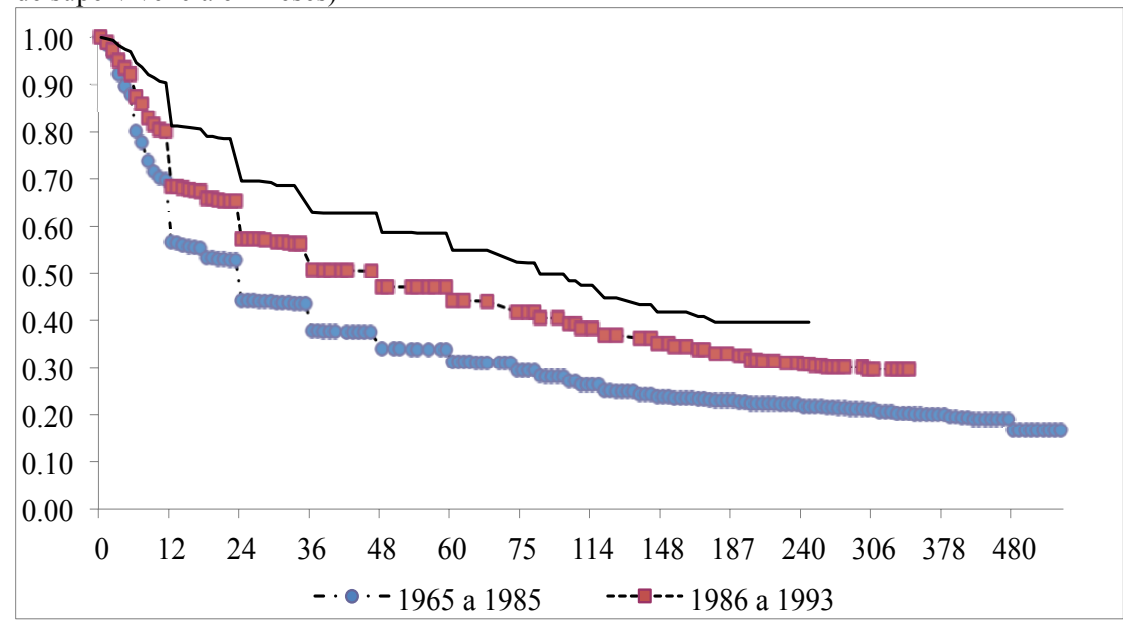

Nota: Se presenta la figura de sobrevivencia en meses del primer viaje migratorio a partir del año1965 en adelante de los migrantes indocumentados.

Fuente: elaboración propia con base en los datos del archivo PERS de la encuesta MMP150.

Tabla 1: Cuartiles del tiempo de permanencia en Estados Unidos de los migrantes indocumentados mexicanos en su primer viaje migratorio por cohorte de salida (meses)

\begin{tabular}{lrr}
\hline Cohorte de salida & Primer cuartil & Mediana \\
\hline 1965 a 1985 & 7 & 23 \\
1986 a 1993 & 11 & 36 \\
1994 a 2013 & 22 & 78 \\
\hline Fuente: elaboración propia con base en los datos del archivo PERS de la encuesta MMP150.
\end{tabular}

Asimismo, los datos muestran que en la cohorte de salida 1965-1985 (periodo indocumentado) el primer cuartil no rebasaba el año de permanencia (siete meses). Sin embargo, para la cohorte de salida 1994-2013 (periodo de la militarización de la frontera) el primer cuartil alcanzó los 22 meses de permanencia; es decir, antes 25 por ciento de los migrantes indocumentados permanecían menos de un año en su primer viaje migratorio en Estados Unidos y ahora 25 por ciento alargan su permanencia a más de un año. Esto muestra un desgaste en la circularidad migratoria, que se refleja en un cambio en el patrón migratorio de temporal a uno de tipo más "permanente" durante el periodo de la política de militarización de la frontera. Esto confirma los resultados de otros autores como Massey et al. (2009), Tuirán y Ávila (2010), Alba (2009), Passel y D’Vera (2009) y Riosmena (2009). 
Además de estos resultados, diferentes encuestas dan cuenta del incremento del tiempo de permanencia de los migrantes indocumentados durante el periodo de reforzamiento de la frontera. Por ejemplo, la información de los flujos de migrantes temporales captada por las EMIF 1993-2003 registran un incremento en el tiempo de estancia y de la disminución del número de personas que se insertan en los circuitos migratorios recurrentes.

Asimismo, con base en las ENADID 1992 y 1997 y en el Censo de Población y Vivienda de México de 2000, se encontró que la duración de la estancia de los migrantes indocumentados ha sido mayor a finales de los años noventa; en 1992, cerca de 20 por ciento de los migrantes mexicanos que se dirigían a Estados Unidos retornaron después de seis meses; en 1997, alrededor de 15 por ciento regresaron a México en el mismo intervalo de tiempo y, para el año 2000, sólo siete por ciento de los migrantes lo hizo (Cornelius, 2007).

Todo lo anterior muestra que el endurecimiento de la política de reforzamiento de la frontera en el periodo post-IRCA ha alargado la estadía en Estados Unidos de los migrantes indocumentados y ha incrementado la probabilidad de asentarse de manera más permanente en Estados Unidos (Tuirán y Ávila, 2010; Tuirán et al., 2000; Massey et al., 2009; 2002; Cornelius, 2007; Riosmena, 2004).

\section{Metodología de estimación}

Para cuantifica el efecto que tienen los costos y riesgos asociados con el cruce fronterizo clandestino sobre el tiempo de permanencia de los migrantes se ajustó un modelo de riesgo en tiempo discreto logit, el cual permite determinar cambios en las probabilidades de retorno y, por lo tanto, cambios en las tendencias de las duraciones de los viajes migratorios.

La base de datos empleada es la Mexican Migration Project (MMP150), debido a que recopila de manera retrospectiva la historia de vida del jefe del hogar (así como de su cónyuge) relacionada a su experiencia migratoria en Estados Unidos y reúne a diferentes niveles de análisis (individual, hogar, localidad, país) información detallada de variables que permite la estimación de modelos de regresión multivariados (Massey, 2006).

La variable que se utiliza para medir el incremento de los riesgos durante el cruce fronterizo clandestino es el Presupuesto Anual de la Patrulla Fronteriza (PAPF), rezagado un año (t-1). Se seleccionó este dato porque los incrementos en el PAPF durante el periodo de militarización de la frontera han sido aprobados para la construcción de muros fronterizos, la contratación de más agentes de la patrulla fronteriza y la compra de avanzados 
equipos de vigilancia, esto con el fin de desviar los flujos migratorios indocumentados hacia zonas rurales cada vez más remotas y peligrosas, lo que ha incrementado los riesgos en el cruce fronterizo clandestino. La variable está rezagada un año debido a que una vez que el congreso americano aprueba el presupuesto de esta dependencia gubernamental, se necesita un periodo de tiempo para la construcción de las barreras físicas. Dicha variable, está definida en el modelo econométrico como: Riesgo de cruce fronterizo. Para los migrantes indocumentados, la variable toma el valor del presupuesto de la patrulla fronteriza rezagado un año en relación al año de cruce, mientras que, para los migrantes documentados la variable toma el valor de cero. En este caso a los migrantes documentados se les asigna el valor de cero debido a que éstos, al poder cruzar la frontera de manera segura, no están afectados por los riesgos de cruce fronterizo clandestino.

En la Figura 4 se muestra el Presupuesto Anual de la Patrulla Fronteriza y se aprecia un incremento a partir de 1986, año en el cual se comienza a configurar la política de reforzamiento de la frontera con IRCA, posteriormente se aprecia un creciente incremento a partir de 1993 lo que marca la implementación de la política de militarización de la frontera, la cual ha incrementado marcadamente los riesgos de cruce fronterizo a los migrantes indocumentados.

Figura 4: Presupuesto anual de la patrulla fronteriza en millones de dólares, 1965 a 2013 (dólares en términos reales a marzo 2014)

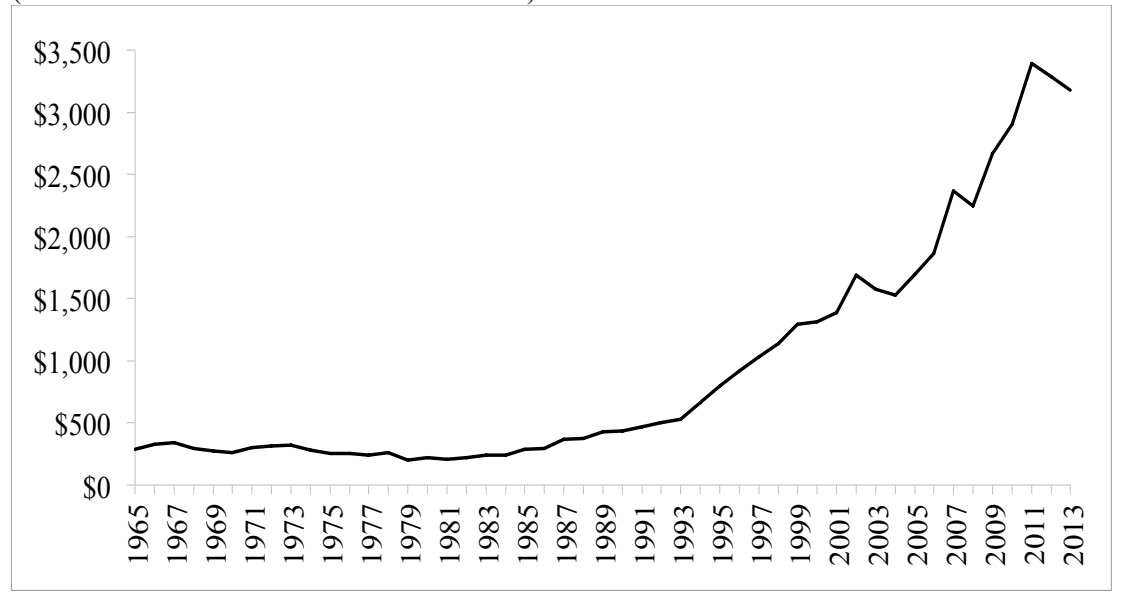

Fuente: elaboración propia con base en los datos de la encuesta MMP150 y de los datos del U.S. Department of Labor: Bureau of Labor Statistics Washington, D.C. Consumer Price Index of All Urban Consumers (CPI-U). 
Para captar el efecto diferenciado que tienen los riesgos y los costos de cruce fronterizo se incluye la variable de costo de cruce en dólares reales (marzo 2014), la cual toma el valor promedio del pago por los servicios del "coyote" en el año en que se efectuó el cruce fronterizo. Para los migrantes documentados, el costo de cruce fronterizo se calculó utilizando los costos en 2013 del trámite de su pasaporte y visa de trabajo, lo cual se estimó en 350 USD; para obtener la estimación de los demás años, se deflactó dicho costo.

Para determinar la manera en la cual el retorno depende del tiempo de exposición, se crearon $n-1$ variables dicotómicas independientes para cada uno de los intervalos de respuesta, los cuales fueron determinados con base en la tabla de sobrevivencia, la cual muestra que en los primeros cuatro semestres $(6,12,18$ y 24 meses) se concentran la mayoría de los retornos. Sin embargo, después de transcurridos los 24 meses, la concentración de los retornos se vuelve anual (36, 48, 60 y 72 meses y más). No se incluye en la regresión la variable dicotómica que agrupa a los primeros seis meses para evitar el problema de multicolinealidad perfecta.

Por otro lado, dado que diferentes trabajos han encontrado evidencia estadística que sustenta que: las característica individuales, hogar, localidad y país afectan a la probabilidad de retorno de los migrantes en Estados Unidos, el modelo econométrico incluye estas dimensiones, asimismo se incluyen una variable de control: lugar en donde se levantó la entrevista (México o Estados Unidos) con el fin de diferenciar a los migrantes temporales de los permanentes.

Las variables que no fueron estadísticamente significativas en el modelo fueron eliminadas del mismo para no perder grados de libertad y por parsimonia (Guillén, 2006). La Tabla 2 resume las variables empleadas en el modelo empírico y muestra la definición operativa empleada para el ajuste del modelo.

\section{INTERPRETACIÓN DE RESULTADOS}

La Tabla 3 muestra los valores de los estimadores de regresión $\left(\beta_{\mathrm{i}}\right)$ del modelo de riesgo logit, el cual presenta una bondad de ajuste de 0.70. En relación con el tiempo de permanencia en Estados Unidos y tomando como grupo de referencia los primeros seis meses de estancia en ese país, la probabilidad de retorno aumenta del séptimo al doceavo mes de estancia en Estados Unidos (segundo semestre), mientras que disminuye en los siguientes periodos de tiempo de permanencia. Una mayor educación así como una mayor experiencia laboral en Estados Unidos disminuyen la 
probabilidad de retorno debido a que permiten el acceso a empleos mejor remunerados, lo cual incentiva el asentamiento de los migrantes en el país de destino. Trabajar en el sector agrícola aumenta la probabilidad de retorno, debido a que en general estos empleos son estacionales, con lo cual los migrantes no pueden hacer frente a los costos fijos que implica una estancia de tipo permanente en Estados Unidos. En cuanto al capital social, si el jefe del hogar migra con su esposa a Estados Unidos, su probabilidad de retorno disminuye.

Los activos del hogar también median en la probabilidad de retorno ya que si el migrante proviene de un hogar que cuenta con tierra cultivable, un negocio o ambos, la probabilidad de retorno aumenta debido a que no necesitan permanecer por largos periodos de tiempo empleado en el mercado laboral de destino con el fin de obtener un nivel de ingresos tal que le permita el financiamiento de estos activos productivos. A un nivel más agregado, se observa que los migrantes que provienen de comunidades con infraestructura productiva, la cual permite tener oportunidades de inversiones rentables, presentan probabilidades de retorno menores, debido a que tienen fuertes incentivos de permanecer por largos periodos de tiempo empleados en el mercado laboral de destino con el fin de obtener un nivel de ingreso que les permita el financiamiento de inversiones en actividades productivas.

Asimismo, como era de esperar los migrantes temporales (entrevistados en México) presentan una mayor probabilidad de retorno.

En cuanto a nuestras variables de interés, el modelo muestra que una vez controlando por las demás variables que median en el tiempo de permanencia, tanto los costos como los riesgos de cruce fronterizo son significativos y con una relación negativa con respecto a la probabilidad de retorno, con lo cual se encuentra evidencia estadística (con un nivel de confianza mayor a 95 por ciento) que señala que cada una de estas variables interviene en la determinación del tiempo de permanencia al disminuir la probabilidad de retorno si los valores de estas variables se incrementan.

Según la teoría Targer Income (Berg, 1961; Hill, 1987) a mayor costo de migrar mayores son los incentivos del migrante de permanecer durante más tiempo en el país receptor. Debido a que para lograr alcanzar su meta de ahorro necesitan trabaja más tiempo en el mercado laboral de ese país, ya que tienen que compensar los mayores costos del cruce fronterizo (Massey et al., 2009; Cornelius, 2001; Lindstrom, 1996). 
Tabla 2: Definición de variables modelo logit (Discrete-Time Hazard Model)

Variable dependiente
Probabilidad de retorno
Variables independientes
Patrón de tiempo de respuesta (semestre)

Primero

Segundo

Tercero

Cuarto

Quinto y sexto

Séptimo y octavo

Noveno y décimo

Onceavo y más

Capital humano general

Educación

Capital humano migratorio

Experiencia laboral en Estados Unidos

Migrante trabaja en el sector agrícola

Conexiones personales del migrante en el hogar de arribo

Esposa migra junto con el esposo a Estados Unidos

Necesidades de la inversión del hogar

Migrante posee tierra cultivable y/o negocio

Oportunidades de inversión en la comunidad

Costo de migrar al país de destino

Costo de cruce fronterizo

Incremento del riesgo en el cruce fronterizo pais de destino

Riesgo de cruce fronterizo

Migración temporal vs permanente

Migrante temporal

Notas: ${ }^{a}$ Adicionalmente a estas variables se probó incluir en el modelo: Sexo, edad, familia posee casa, número de dependientes en el hogar, número de hijos nacidos en Estados Unidos y población rural-urbana. Estas variables no fueron estadísticamente significativas, por lo que se eliminaron del modelo para no perder grados de libertad y por parsimonia. ${ }^{b}$ Los intervalos del tiempo se determinaron con base en la tabla de sobrevivencia. Fuente: elaboración propia con base en los modelos de: Massey y Espinosa, 1997; Massey et al., 1987; Lindstrom, 1996.

\section{Definición operativa}

Dummy $=1$ si se regresa en el semestre de observación; 0 en otro caso

1 si el viaje migratorio ha durado 6 meses y 0 en otro caso

1 si el viaje migratorio ha durado entre 7 y 12 meses y 0 en otro caso

1 si el viaje migratorio ha durado entre 13 y 18 meses y 0 en otro caso

1 si el viaje migratorio ha durado entre 19 y 24 meses y 0 en otro caso

1 si el viaje migratorio ha durado entre 25 y 36 meses y 0 en otro caso

1 si el viaje migratorio ha durado entre 37 y 48 meses y 0 en otro caso

1 si el viaje migratorio ha durado entre 49 y 60 meses y 0 en otro caso

1 si el viaje migratorio ha durado entre 61 y más meses y 0 en otro caso

Número de años de educación

Meses de experiencia migratoria acumulada en Estados Unidos

1 si en el viaje migratorio trabaja en el sector agrícola de Estados Unidos y 0 en otro caso

1 si la esposa migra junto con el esposo a Estados Unidos y 0 en otro caso

1 si la familia posee tierra cultivable o negocio en México y 0 en otro caso

Número de sucursales bancarias en el municipio

Pago por cruzar la frontera en dólares reales a marzo de 214

Presupuesto anual de la patrulla fronteriza rezagado al año de cruce $(\mathrm{t}-1)$ 1 para encuesta levantada en México y 0 en otro caso 
Tabla 3: Valores de los estimadores de regresión $\left(\beta_{\mathrm{i}}\right)$ del modelo logístico de historia de eventos

\begin{tabular}{lrrr}
\hline Variables & Coeficiente & $\mathrm{z}$ & $\mathrm{p}>|\mathrm{z}|$ \\
\hline Constante del modelo & -0.6026 & -9.19 & 0.000 \\
Segundo semestre & 0.2551 & 6.41 & 0.000 \\
Tercer semestre & -1.3324 & -20.29 & 0.000 \\
Cuarto semestre & -0.4430 & -9.46 & 0.000 \\
Quinto y Sexto semestre & -0.4416 & -7.72 & 0.000 \\
Séptimo y Octavo semestre & -0.5159 & -7.29 & 0.000 \\
Noveno y Decimo semestre & -0.5232 & -6.19 & 0.000 \\
Onceavo semestre y más & -0.4331 & -7.29 & 0.000 \\
Educación & -0.0233 & -5.96 & 0.000 \\
Experiencia laboral en Estados Unidos & -0.0125 & -45.24 & 0.000 \\
Migrante trabaja en el sector agrícola & 0.6568 & 19.54 & 0.000 \\
Esposa migra junto con el esposo a Estados Unidos & -0.7173 & -13.70 & 0.000 \\
Migrante posee tierra cultivable y/o negocio & 0.2441 & 6.35 & 0.000 \\
Oportunidades de inversión en la comunidad & -0.0043 & -4.10 & 0.000 \\
Costo de cruce fronterizo & -0.0001 & -3.40 & 0.000 \\
Riesgo de cruce fronterizo & -0.0006 & -16.30 & 0.000 \\
Migrante temporal & 0.9289 & 18.71 & 0.000 \\
\hline
\end{tabular}

Nota: El nivel de significancia de todos los regresores es de $p<0.05$.

Fuente: elaboración propia con base en los datos de la encuesta MMP150.

Por ejemplo, con un trabajo de 40 horas a la semana, a cinco dólares la hora, los migrantes pueden pagar el costo del "coyote" de 500 dólares en 2.5 semanas, pero con los costos actuales de 2850 dólares los migrantes tienen que aumentar el número de semanas trabajadas a 14.25 (Massey et al., 2009).

En cuanto a los riesgos de cruce fronterizo dado que el reforzamiento de la frontera ha venido incrementándolos a un grado, en el cual incluso los migrantes se exponen a perder la vida, es de esperar que éstos prefieran extender su estancia al máximo o bien asentarse de manera más permanente en Estados Unidos, ya que no quieren repetir otra vez la amarga y peligrosa experiencia de transitar por la frontera bajo esas condiciones de cruce.

\section{Estimación de la probabilidad de retorno}

Para calcular el efecto que tiene el aumento de los riesgos asociados al cruce fronterizo clandestino sobre la probabilidad de retorno se sustituye los valores del Presupuesto Anual de la Patulla Fronteriza (PAPF) en la función de probabilidad logit, manteniendo todas las demás variables fijas. Para esto utilizamos los valores de las variables explicativas $\left(\mathrm{x}_{\mathrm{i}}\right)$ que describen un ejemplo de un migrante con características que se asocian con 
un patrón migratorio de tipo temporal, es decir; la persona tiene una baja educación, trabaja en el sector agrícola de Estados Unidos, su esposa no ha migrado, posee tierra cultivable y/o negocio en México, proviene de una comunidad que permite limitadas oportunidades de inversión, su experiencia laboral en Estados Unidos es de 36 meses y dado que no tiene documentos sus costos de cruce fronterizo en un escenario de reforzamiento de la frontera es de 3000 USD. Además, es un migrante que ha permanecido en Estados Unidos por doce meses. La Tabla 4 resume estos valores.

Sustituyendo en la función de probabilidad del modelo logit los coeficientes de regresión $\left(\beta_{\mathrm{i}}\right)$, así como los valores de las variables explicativas $\left(\mathrm{x}_{\mathrm{i}}\right)$ y utilizando el presupuesto anual de la patrulla fronteriza (PAPF) como medida del incremento en los riesgos asociados al cruce fronterizo clandestino se obtiene la probabilidad que tiene un migrante temporal indocumentado de retornar ante el aumento de los costos y riesgos asociados al cruce fronterizo clandestino que surgen de la política migratoria de reforzamiento de la frontera (Figura 5).

La Figura 5 muestra que una vez controlado los altos costos que se pagan (3 000 USD) por cruzar la frontera de manera indocumentada (los cuales surgen por el reforzamiento de la frontera), así como por las demás variables, la probabilidad de retorno a los doce meses de permanencia para una persona con un perfil migratorio de tipo temporal variaba entre $0.60 \mathrm{y}$ 0.58, durante el periodo de 1965 a 1985, durante el cual el PAPF fluctuaba entre 200 y 338 millones de dólares. Posteriormente a partir de 1986 con el incremento gradual en los riesgos asociados al cruce fronterizo clandestino (PAPF) que se registra durante el periodo IRCA, la probabilidad de retorno a los doce meses disminuyó ligeramente en el tiempo, al pasar de 0.59 en 1986 (PAPF 296.11 millones de dólares) a 0.56 en 1993 (PAPF 526.32 millones de dólares). Sin embargo es a partir de la implementación de la política de militarización de la frontera que la probabilidad de retorno ha caído marcadamente en el tiempo, al pasar de 0.56 en 1993 (PAPF 526.32 MD) a sólo 0.16 en 2013 (PAPF 3179.84 MD), con lo cual es más probable que los migrantes indocumentados permanezcan en Estados Unidos en vez de retornar a sus comunidades de origen una vez cumplido los doce meses de viaje. Estos cambios en las probabilidades de retorno a partir de 1993, muestran que el aumento en los costos y riesgos de la política de militarización de la frontera ha provocado que los migrantes alarguen su permanencia en Estados Unidos. 


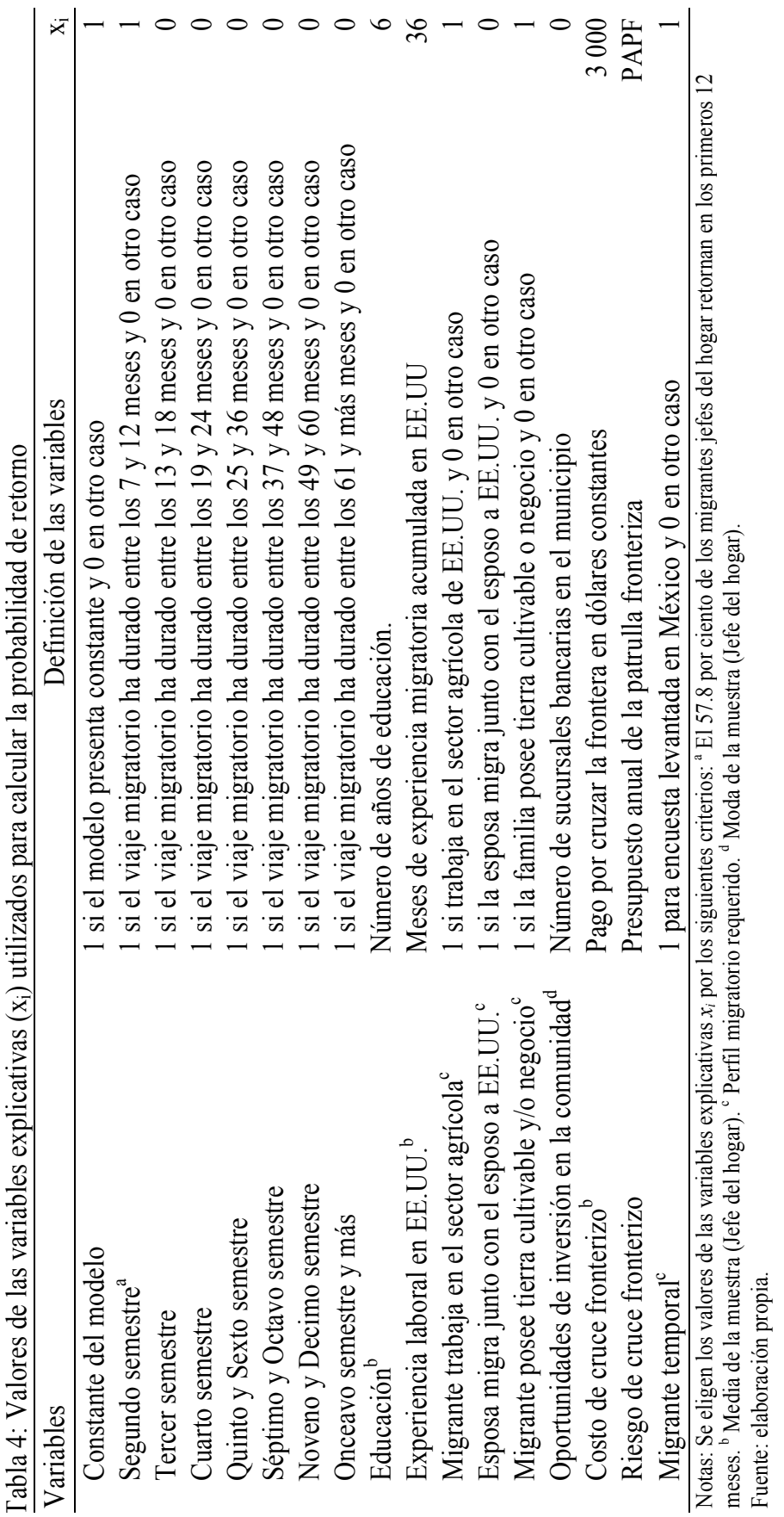


Figura 5: Probabilidad de retorno de los migrantes indocumentados en los primeros doce meses de viaje, ante el incremento en los riesgos asociados al cruce fronterizo clandestino (PAPF de 1965 a 2013), controlando por los elevados costos de cruce clandestino

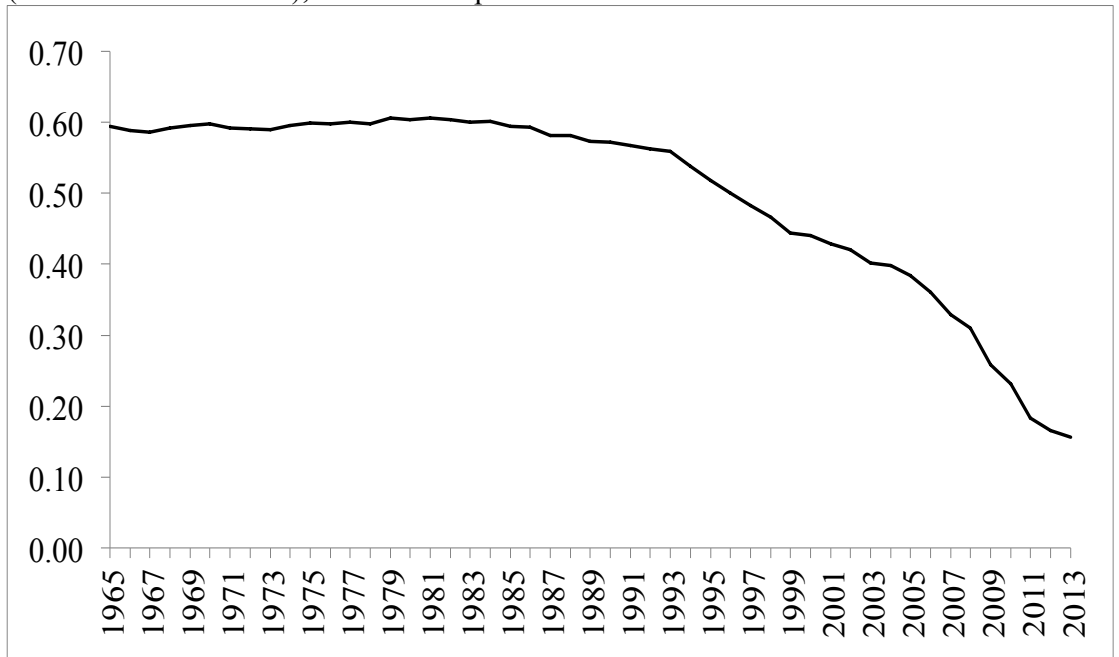

Nota: Se suavizan (siguiendo su tendencia) los valores del presupuesto anual de la patrulla fronteriza de los años 2002, 2007 y 2011.

Fuente: elaboración propia, con base en los valores resumidos en la Tabla $3\left(\beta_{\mathrm{i}}\right)$ y en la Tabla $4\left(\mathrm{x}_{\mathrm{i}}\right)$ del presupuesto anual de la patrulla fronteriza (PAPF) como medida del incremento en los riesgos asociados al cruce fronterizo clandestino.

\section{Conclusiones}

La migración México-Estados Unidos tiene una larga historia que puede ser dividida en fases o eras que se distinguen por las características del cruce fronterizo y las condiciones legales que prevalecen. La era migratoria indocumentada (1965-1985) que comenzó con la cancelación del programa bracero en 1964, se caracterizó por la circularidad migratoria de personas indocumentadas debido, en parte, a la relativa facilidad con la que se podía cruzar la frontera México-Estados Unidos Sin embargo, es a partir de la promulgación de la ley IRCA en 1986, y del lanzamiento de la política de reforzamiento de la frontera en 1993, que se comienza a escribir una nueva era migratoria, la cual se caracteriza por tratar de controlar los cruces fronterizos de personas no autorizadas (Massey et al., 2009; Durand y Massey, 2009). En relación a este nuevo ciclo migratorio, se ha observado un marcado desgaste de los mecanismos de circularidad migratoria y una mayor propensión a una migración de carácter más permanente. Esto 
se explica, en parte, ${ }^{25}$ porque la política de reforzamiento de la frontera ha aumentado los costos y los riesgos en el cruce fronterizo clandestino.

Acorde con lo anterior, el análisis de sobrevivencia de los migrantes indocumentados mexicanos en su primer viaje migratorio durante nuestro periodo de estudio (1965-2013), muestra que en la cohorte de salida 19651985 (periodo indocumentado) presenta siete meses de permanencia en el primer cuartil, mientras que en la cohorte de salida de 1986-1993 (periodo post-IRCA) el tiempo de permanencia del primer cuartil aumentó a 11 meses. Para la cohorte de salida 1994-2013 (periodo del reforzamiento de la frontera), el primer cuartil alcanzó los 22 meses de permanencia. Es decir antes de la implementación de la política de reforzamiento de la frontera en 1993, 25 por ciento de los migrantes indocumentados permanecía menos de un año en su primer viaje migratorio en Estados Unidos y, posterior a ésta, se alarga la permanencia de este 25 por ciento a más de un año. Lo anterior muestra que la implementación de la política de reforzamiento de la frontera en el periodo post-IRCA ha disminuido la probabilidad de retorno, desgastando la circularidad migratoria, que se refleja en un cambio en el patrón migratorio de temporal a uno de tipo más "permanente".

Además, los resultados del modelo muestran que tanto las variables de costos como riesgos de cruce fronterizo son estadísticamente significativos con un nivel de confianza de 95 por ciento y con una relación negativa con respecto a la probabilidad de retorno, con lo cual se encuentra evidencia estadística consistente que señala que cada una de estas variables interviene en la determinación del tiempo de permanencia al disminuir la probabilidad de retorno si los valores de estas variables aumentan. Estos resultados respaldan la literatura (target income) que señala que si los costos de migrar se incrementan, los migrantes deben de permanecer por más tiempo trabajando en el país de destino con el fin de recuperar dichos costos. Mientras que, por el lado de los riesgos, los resultados del modelo estadístico señalan que al aumentar los riesgos de cruce fronterizo clandestino los migrantes optarán por alargar su tiempo de permanencia ya que no quieren exponerse nuevamente a la peligrosa experiencia de cruzar la frontera.

Por el lado de la estimación de la probabilidad de retorno, los resultados muestran que una vez controlando por las demás variables -incluidos los altos costos de cruzar la frontera - el aumento de los riesgos en el cruce fronterizo clandestino producto del reforzamiento de la frontera ha

\footnotetext{
${ }^{25}$ Aunado a los costos y riesgos del cruce fronterizo clandestino, otras variables tales como una migración de tipo familiar (esposa migra junto con su esposo), inserción laboral en trabajos más permanentes (no agrícolas), migrantes mejor calificados (migrantes con más años de educación), entre otras, median en el alargamiento de la permanencia de los migrantes en Estados Unidos.
} 
disminuido la probabilidad de retorno a los 12 meses de haber ingresado a Estados Unidos para los migrantes con un perfil que tradicionalmente se pensaba como temporal. Este perfil se asociaba con trabajadores en el sector agrícola de Estados Unidos, viajando solos, que poseían tierra cultivable y/o negocio en México, de comunidades con pocas oportunidades de inversión, y con baja escolaridad. Los datos muestran que, antes de la implementación en 1993 de esta política migratoria, la probabilidad de retorno en los primeros doce meses de viaje de una persona con el perfil mencionado oscilaba entre 0.61 y 0.56 ; en contraste, a partir de la puesta en marcha de esta política y de su endurecimiento en los subsiguientes años, lo cual ha incrementado a través del tiempo los riesgos de cruce fronterizo clandestino, la probabilidad de retorno para una persona con este perfil cayó constantemente hasta llegar a sólo 0.16 en 2013.

En la actualidad los datos del Censo de 2010 levantado en México muestran que el aumento del retorno a México no fue alto en términos relativos (aunque si en términos de volumen). Esto resulta contradictorio si consideramos la contracción económica en los sectores productivos de Estados Unidos en los cuales los migrantes se emplean. Esto sugiere que los altos costos y riesgos en el cruce fronterizo clandestino desincentivan a los migrantes a retornar a su comunidad de origen, a pesar de la coyuntura económica desfavorable que deben de vivir los migrantes en aquel país. Dicho de otro modo, el retorno de los migrantes a México se presenta únicamente cuando se han agotado todas las opciones que permitan permanecer en territorio estadounidense a pesar de la recesión, debido a los altos costos de cruce fronterizo y a que los migrantes no quieren repetir la dura y amarga experiencia de volver a cruzar la frontera de manera clandestina bajo las condiciones que imperan en la actualidad.

Los resultados del modelo sugiere que una de las causas por las que se registra en la actualidad un aumento en los retornos es debido a que se han incrementado de manera significativa las deportaciones por parte del $\mathrm{De}$ partment of Homeland Security (DHS) de migrantes mexicanos que viven y trabajan en Estados Unidos de manera indocumentada; ya que durante el periodo de 2004 a 2013 se registraron un total de 2409967 deportaciones.

Todo lo anterior apunta a que en la actualidad el patrón de permanencia de los migrantes indocumentados sigue siendo el de alargar su estancia en Estados Unidos pero muchos de ellos son obligados a retornar a México ya que son removidos del territorio de Estados Unidos, lo cual sobreestima la cifra de las personas que quieren retornar a México de manera voluntaria y planeada. 
Una pregunta de investigación que abre este estudio es: ¿Como podemos identificar y explicar los mecanismos a través de los cuales el aumento en los riesgo asociados al cruce fronterizo clandestino afecta per se al tiempo de permanencia de los migrantes indocumentados?, ya que el estudio sólo encuentra evidencia estadística de la relación que guardan estas dos variables.

\section{REFERENCIAS BIBLIOGRÁFICAS}

Alba, Francisco, 2010, "Respuestas mexicanas frente a la migración a Estados Unidos", en Los grandes problemas de México: migraciones internacionales, El Colegio de México.

Alba, Francisco, 2009, "Migración internacional y políticas públicas", en El estado de la migración: las políticas públicas ante los retos de la migración mexicana a Estados Unidos. Consejo Nacional de Población.

Alba, Francisco, 2007, "La reconsideración de la política migratoria internacional", en México: los retos ante el futuro, El Colegio de México.

Allison, Paul D., 1984, Event History Analysis: Regression for Longitudinal Event Data. Sage University Papers.

Allison, Paul D., 1982, "Discrete-Time methods for the analysis of event histories", in Sociological Methodology, vol. 13.

Andreas, Peter, 2000, Border games: policing the U.S.-Mexico Divide. Cornell University Press.

Andreas, Peter, 1999, "The escalation of U.S. immigration control in the post-NAFTA era", in Political Science Quarterly, vol. 113, No. 4, Winter.

Ariza, Marina y Portes, Alejandro, 2010, "La migración internacional de mexicanos: escenarios y desafíos de cara al nuevo siglo", en El país transnacional: migración mexicana y cambio social a través de la frontera. Miguel Ángel Porrúa.

Berg, Elliot J., 1961, "Backward-Sloping labor supply functions in dual economies: the Africa case", in Quarterly Journal of Economics, vol. 75, No. 3.

Cornelius, Wayne A., Borger, Scott, Sawyer, Adam, Keyes, David, Appleby, Clare, Parks, Kristen, Lozada, Gabriel and Hicken, Jonathan, 2008, Controlling unauthorized immigration from Mexico: the failure of "Prevention through Deterren$c e$ " and the need for comprehensive reform, Center for Comparative Immigration Studies (CCIS), University of California-San Diego.

Cornelius, Wayne A., Borger, Scott, Sawyer, Adam, Keyes, David, Appleby, Clare, Parks, Kristen, Lozada, Gabriel and Hicken, Jonathan, 2007, "Does border enforcement deter unauthorized immigrant?", in Impacts of border enforcement on mexican migration: the view from sending communities, Center for Comparative Immigration Studies, UCSD. 


\section{Duración de los viajes migratorios de mexicanos indocumentados en Estados Unidos bajo ... / R. MORA TÉLLEZ}

Cornelius, Wayne A., Borger, Scott, Sawyer, Adam, Keyes, David, Appleby, Clare, Parks, Kristen, Lozada, Gabriel and Hicken, Jonathan, 2001, "Death at the border: efficacy and unintended consequences of US immigration control policy", in Population and Development Review, vol. 27, No. 4.

Department of Homeland Security, 2013, Immigration Enforcement Actions: Annual Report.

Dunn, Timothy J., 1997, The militarizing of the U.S.-Mexico border, 1978-1992: low-intensity conflict doctrine comes home, Center for Mexican American Studies -University of Texas, Austin.

Durand, Jorge y Massey, Douglas S., 2009, Clandestinos migración México-Estados Unidos en los albores del siglo XXI, Miguel Ángel Porrúa.

Durand, Jorge y Massey, Douglas, S., 1994, Más allá de la línea: patrones migratorios entre México y Estados Unidos. Consejo Nacional para la Cultura y las Artes. México.

Eschbach, Karl, Hagan, Jacqueline and Rodríguez, Néstor, 2001, Causes and trends in migrant deaths along the U.S.-Mexico border, 1985-1998, Center for Immigration Research, University of Houston,

Eschbach, Karl, Hagan, Jacqueline and Rodríguez, Néstor, Hernandez-Leon, Rubén and Bailey, Stanley, 1999, "Death at the border", in International Migration Review. vol. 33, No. 2.

Espenshade, Thomas J., 1994, "Does the threat of apprehension deter undocumented U.S. immigration?", in Population and Development Review, vol. 20, No. 4.

Espenshade, Thomas J., 1990, "Undocumented migration to the United States: Evidence from a repeated trial model", in Undocumented Migration to the United States: IRCA and the Experience of the 1980s, Urban Institute Press, Washington, D. C.

General Accounting Office, 2006, Illegal immigration: border-crossing deaths have double since 1995; Border patrol's efforts to prevent deaths have not been fully evaluated. August, GAO-06-770.

Guillén Fernández, Y. Betzabé, 2006, Factores demográficos y socioeconómicos asociados a la desnutrición infantil en México a principios del siglo XXI, El Colegio de México.

Hill, John K., 1987, "Immigrant decisions concerning duration of stay and migratory frequency", in Journal of Development Economics, vol. 25, issue 1.

Izacara Palacios, Simón Pedro, 2014, "La transformación del coyotaje en el contexto de políticas migratorias restrictivas", en Estudios Sociales, vol. 23, núm. 46.

Kossoudji, Sherrie A., 1992, "Playing cat and mouse at the U.S.-Mexican border", in Demography, vol. 29, No. 2.

Lindstrom, David P., 1996, "Economic opportunities in Mexico and return migration from the United States", in Demography, vol. 33, No. 3. 
Massey, Douglas S., 2014, Pasado y Futuro de la Migración Indocumentada en Norteamérica, conferencia dictada en el Instituto de Investigaciones Sociales de la Universidad Nacional Autónoma de México (IIS-UNAM), 27 de Mayo de 2014.

Massey, Douglas S. and Riosmena, Fernando, 2010, "Undocumented migration from latin American in an era of rising U.S. enforcement", in The ANNALS of the American Academy of Political and Social Science.

Massey, Douglas S., Pren, Karen A. y Durand, Jorge, 2009, "Nuevos escenarios de la migración México-Estados Unidos: Las consecuencias de la guerra antiinmigrante", en Papeles de Población, vol. 15, núm. 61.

Massey, Douglas S. y Chiara, Capoferro, 2006, "La medición de la migración indocumentada", en Repensando las migraciones: nuevas perspectivas teóricas y empíricas. Instituto Nacional de Migración.

Massey, Douglas S., Durand, Jorge and Malone, Nolan J., 2002, Beyond smoke and nirrors: Mexican immigration in an era of economic integration. Russell Sage Foundation, New York.

Massey, Douglas S. and Espinosa, Kristin, 1997, "What's driving Mexico-U.S. migration? A theoretical, empirical, and policy analysis", in American Journal of Sociology, vol. 102, No. 4.

Massey, Douglas S. and Liang, Zai, 1989, "The long-term consequences of a temporary worker program: The U.S. bracero experience", in Population Research and Policy Review, vol. 8, No. 3.

Massey, Douglas S., 1987, "Understanding Mexican migration to the United States", in The American Journal of Sociology, vol. 92, No. 6.

Nagengast, Carol, 1998, "Militarizing the border patrol", in NACLA, vol. 32. Nueva York.

Orrenius, Pia M. and Zavodny, Madeline, 2005, "Self-selection among undocumented immigrants from Mexico", in Journal of Development Economics, vol. 78 , issue 1 .

Orrenius, Pia M. and Zavodny, Madeline, 2004, "The effect of U.S. border enforcement on the crossing behavior of mexican migrants", in Crossing the border: research from the mexican migration project. Russell Sage Foundation. New York.

OIM, 2014, Fatal journeys. tracking lives lost during migration, Organización Internacional para las Migraciones (OIM), Geneva, Switzerland.

Passel, Jeffrey, Cohn, D’Vera and González-Barrera, Ana, 2012, "Net migration from Mexico falls to zero- and perhaps less", in Pew Hispanic Center, april 23.

Passel, Jeffrey and Cohn, D’Vera, 2011, “Unauthorized immigrant population: National and sate trends 2010", in Pew Hispanic Center, february 1.

Passel, Jeffrey and Cohn, D'Vera, 2009, "Mexican immigrants: how many come? how many leave?", in Pew Hispanic Center, july 22. 
Richter, M. Susan, J. Taylor, Edward and Yúnez-Naude, Antonio, 2007, "Impacts of policy reforms on labor migration from rural Mexico to the United States", in Mexican immigration to the United States. National Bureau of Economic Research, The University of Chicago Press.

Tuirán Rodolfo y Ávila, José Luis, 2010 "La migración México-Estados Unidos, 1940-2010", en Los Grandes Problemas de México: Migraciones Internacionales, El Colegio de México.

Tuirán Rodolfo, Fuentes, Carlos y Ávila, José Luis, 2000, Índice de intensidad migratoria México-Estados Unidos 2000. Consejo Nacional de Población, México.

\section{RESUMEN CURRICULAR DEL AUTOR}

\section{Ricardo Mora Téllez}

Cursó la maestría en Economía en el Centro de Investigación y Docencias Económicas (CIDE), además recibió su grado de Doctor en Demografía por el Colegio de México. Cuenta con una estancia de investigación en la Universidad de Princeton durante la cual se incorporó al equipo de investigadores del Proyecto sobre Migración Mexicana (MMP). Ha trabajado en la Cámara de Diputados en las comisiones de Desarrollo Social y Salud y en el Consejo Nacional de Población (CONAPO) en el área de Migración Internacional.

Dirección electrónica: rmora@colmex.mx

Artículo recibido el 30 de noviembre de 2015 y aprobado el 17 de abril de 2017. 